\title{
Tree shrew as a new animal model for the study of lung cancer
}

\author{
LIANHUA YE, MENG HE, YUNCHAO HUANG, GUANGQIANG ZHAO, \\ YUJIE LEI, YONGCHUN ZHOU and XIAOBO CHEN
}

\begin{abstract}
Department of Thoracic Surgery of the Third Affiliated Hospital to Kunming Medical University, Tumor Hospital of Yunnan Province, Kunming, Yunnan 650118, P.R. China
\end{abstract}

Received September 7, 2015; Accepted January 27, 2016

DOI: $10.3892 / \mathrm{ol} .2016 .4156$

\begin{abstract}
Animal models play a key role in identifying treatments for various types of cancer, including lung cancer. The aim of the present study was to develop a new animal model for lung cancer induction using tree shrews from the Yunnan region in China. Tree shrews are suitable for a full simulation of human disease because their structure, function and metabolism are adequately close to human. This animal may offer a new experimental animal model to be used in the study of lung cancer. In the present study, 80 healthy tree shrews were distributed in experimental and control groups. Animals in the experimental group received different concentrations of iodized oil suspension of 3-methylcholanthrene (3-MC) and diethylnitrosamine (DEN) while animals in the control groups received saline or lipiodol solvent via endotracheal instillation. In the $3 \mathrm{rd}, 5$ th, 7th, 9th and 11th weeks the body weights of the animals were measured and chest $\mathrm{X}$-ray examinations were conducted. Pathological studies on the lung tissues were also performed and the pathological changes occurring in bronchial epithelium in all the groups were examined. Animals in the experimental group gradually lost their body weight. For tree shrews in the blank control and solvent control groups the survival rates were 100 and $80 \%$, respectively while the survival rate for the experimental group was $0 \%$. Results from the chest X-ray conducted on animals in the blank control and solvent control groups revealed no obvious abnormalities while in the experimental group high-density shadow spots within the perfusion sites were observed. Pathological studies performed on these high-density areas confirmed changes in the bronchial epithelium. In the experimental groups we also detected bronchial epithelial atypical hyperplasia, and
\end{abstract}

Correspondence to: Drs Lianhua Ye or Yunchao Huang, Department of Thoracic Surgery of the Third Affiliated Hospital to Kunming Medical University, Tumor Hospital of Yunnan Province, 191 Renmin West Road, Kunming, Yunnan 650118, P.R. China E-mail: 1hye1204@aliyun.com

E-mail: huangych2001@aliyun.com

Key words: tree shrew, lung cancer, 3-methylcholanthrene, animal model, diethylnitrosamine apparent changes in carcinoma in situ. In conclusion, lung cancer was successfully induced in tree shrews by a one-time endotracheal introduction of iodized oil suspension of 3-MC and DEN.

\section{Introduction}

In basic and applied research, related to lung cancer pathogenesis and antitumor drugs, animal models have a central role $(1,2)$. Mouse, rat, hamster and rabbit and other rodents have been used to establish a variety of animal models in the study of lung cancer (1-3). However, these animals are not similar to human and are different with regard to living environment, and social psychological status (3). Tupaia belangeri, the tree shrew from the Yunnan province in China, is a fast breeding, small animal that is easy to domesticate and simple to breed. The metabolic and anatomic structure of the tree shrew is closer to human than the dog, rat and other animal models, and has a unique advantage as an animal model for human diseases (4-9).

Previous studies showed that the rat lung cancer model established using 3-methylcholanthrene (3-MC) and diethylnitrosamine (DEN) provided investigators with a better description of gene modification in precancerous and invasive lesions of human lung cancer $(10,11)$.

In the present study, the tree shrew was selected as the animal model due to its resemblance with regard to human structure, function and metabolism. Lung cancer was induced in tree shrews with one-time instillation of the iodized oil suspension of 3-MC and DEN. Through observation of the tree shrews survival and tumor formation rates, we selected the best experimental conditions for developing the best tree shrew lung cancer model, to provide a new type of experimental animal model for the study of lung cancer.

\section{Materials and methods}

Experimental materials. Tree shrews from the Yunnan region were selected for the present study. A total of 80 healthy specific pathogen-free sub-generation tree shrews were provided by Kunming Institute of Zoology of the Chinese Academy of Sciences (Kunming, China). There were 40 female and 40 male animals, with an average age of 6 months \pm 7 days, and a weight of 120-160 g. In addition, 3-MC (Sigma, St. Louis, MO, USA); DEN (Sigma); and iodized oil injection 
Table I. Components dosage form of $0.1 \mathrm{ml}$ lipiodol suspension in each experimental group.

\begin{tabular}{lccc}
\hline Experimental group & 3-Methylcholanthrene, $\mathrm{mg}$ & Diethylnitrosamine, $\mathrm{ml}$ & Iodized oil, $\mathrm{ml}$ \\
\hline 1 & 10 & 0.01 & 0.09 \\
2 & 20 & 0.01 & 0.09 \\
3 & 10 & 0.02 & 0.08 \\
4 & 20 & 0.02 & 0.08 \\
\hline
\end{tabular}

Table II. Comparison of weight changes in tree shrews at different time points in the experimental and control groups ( $g$, mean \pm standard deviation).

\begin{tabular}{lccc}
\hline $\begin{array}{l}\text { Experimental } \\
\text { grouping }\end{array}$ & $\begin{array}{c}\text { Control } \\
\text { group }\end{array}$ & $\begin{array}{c}\text { Experimental } \\
\text { group }\end{array}$ & P-value \\
\hline 1 week & $146.00 \pm 5.78$ & $147.55 \pm 12.56$ & $\mathrm{P}>0.05$ \\
3 week & $148.00 \pm 4.70$ & $135.45 \pm 11.34$ & $\mathrm{P}<0.05$ \\
5 week & $147.00 \pm 8.04$ & $134.23 \pm 12.26$ & $\mathrm{P}<0.05$ \\
7 week & $149.00 \pm 7.67$ & $133.45 \pm 13.17$ & $\mathrm{P}<0.05$ \\
9 week & $152.00 \pm 8.72$ & $132.67 \pm 12.78$ & $\mathrm{P}<0.05$ \\
11 week & $148.00 \pm 6.58$ & $130.34 \pm 11.78$ & $\mathrm{P}<0.05$ \\
\hline
\end{tabular}

Table III. Comparison of the survival rates for tree shrews in the experimental and control groups.

\begin{tabular}{lccr}
\hline Group & $\begin{array}{c}\text { Quantity for } \\
\text { experiment, no. }\end{array}$ & $\begin{array}{c}\text { Surviving } \\
\text { quantity, no. }\end{array}$ & $\begin{array}{c}\text { Survival } \\
\text { rate, \% }\end{array}$ \\
\hline Blank control & 10 & 10 & 100.0 \\
Solvent control & 10 & 8 & 80.0 \\
Experimental & 60 & 23 & 38.3 \\
\hline
\end{tabular}

(Shanhai Xudong Haipu Pharmaceutical Co. Ltd., Shanghai, China) were purchased for the present study.

\section{Experimental method}

Experiment and animal grouping. Experimental groups were set-up and exposed to four different concentrations of iodized oil suspension of 3-MC and DEN (Table I). The blank control group received an equal volume of normal saline, and the solvent control group received lipiodol solvent. The experiments were divided into six groups. Eighty tree shrews were randomly divided into six groups. Each of the four experimental groups had 15 animals, and the two control groups had 10 animals each. During the experiment the tree shrews were kept in a laboratory animal environment of specific pathogen-free at room temperature $\left(20-25^{\circ} \mathrm{C}\right)$ and received adequate amounts of food and water.

Replicate animal model. After the injection of ketamine and atropine, the skin in the neck region was cut, and thyroid cartilage was exposed. In the upper part of the thyroid cartilage, a special needle (comprised of $1 \mathrm{ml}$ syringe needle, designed to be $30^{\circ}$ ) was punctured into the left inferior pulmonary lobe. Tree shrews in each group received a one-time infusion of $0.1 \mathrm{ml}$ iodized oil suspension, saline or iodized oil solvent containing different doses of 3-MC and DEN. Doses received by animals in each experimental group are shown in Table I.

Experimental animal exclusion criteria. Animals did not become ill and had no infectious or non-infectious diseases during the course of the experiment.

General observation of tree shrews. Animal weights in the experimental groups were measured in the 1st, 3rd, 5th, 7th, 9 th and 11th weeks. The activity and survival of tree shrews were monitored closely.

Lung imaging of tree shrews. Tree shrews in the experimental groups underwent X-ray examinations in the 3rd, 5th, 7th, 9th and 11th weeks. Radiographic changes within the chest area were recorded.

Tumor detection of tree shrews. Tree shrews in the experimental groups were sacrificed by cervical dislocation and lung tissue pathology studies were performed in the 3rd, 5th, 7th, 9th and 11th weeks. Light microscope (ECLIPSE E800, Nikon Corporation, Japan) hematoxylin and eosin (H\&E) staining was used to visualize bronchial epithelial pathological changes and tumors.

The present study was approved by the institutional research ethics committee of The Tumor Hospital of Yunnan Province (no. ky201110).

Statistical analysis. The SPSS 17.0 software package (SPSS, Inc., Chicago, IL, USA) was used for statistical analyses. The results were presented as mean \pm standard deviation. The paired t-test was used to pair the mean number of the design data. Skewed distribution data were assessed by the rank sum test. $\mathrm{P}<0.05$ was considered to indicate a statistically significant difference.

\section{Results}

General. During the experiment, the body weights of the tree shrews in the control group did not change significantly, whereas in the experimental group the body weights decreased gradually. Weight measurement comparisons between the two groups in the 5th, 7th, 9th and 11th weeks demonstrated statistically significant differences $(\mathrm{P}<0.05)$ (Table II).

The survival rate of the tree shrews in the blank control group until the end of the experiment was $100 \%$. In the solvent 
Table IV. Comparison of the survival rates for tree shrews with different doses of drug perfusions in each experimental group.

\begin{tabular}{lcrr}
\hline Experimental group & Quantity for experiment, no. & Surviving quantity, no. & Survival rate, $\%$ \\
\hline 1 & 15 & 14 & 93.3 \\
2 & 15 & 5 & 33.3 \\
3 & 15 & 4 & 26.7 \\
4 & 15 & 0 & 0.0 \\
\hline
\end{tabular}
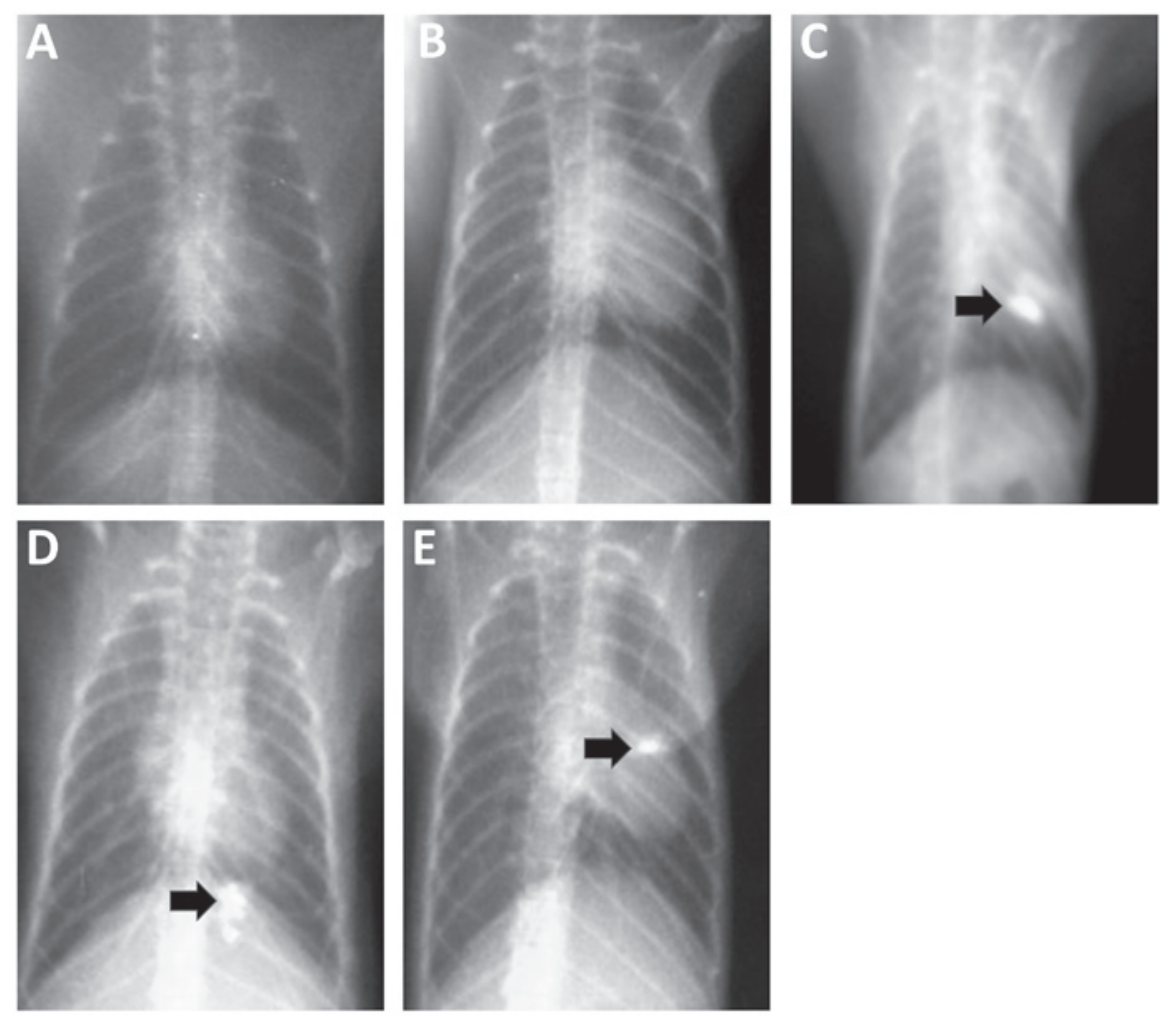

Figure 1. Chest X-rays showing changes in the lungs of tree shrews. (A) Eleventh week X-ray image from an animal in the blank control group. (B) Eleventh week X-ray image from an animal in the solvent control group; (C) third week X-ray image from an animal in the experimental group. (D) Seventh week X-ray image from an animal in the experimental group. (E) Eleventh week X-ray image from an animal in the experimental group.

control group, 8 of 10 animals (80\% survival rate). The death of the two tree shrews (20\%) was confirmed to be associated with puncture-induced hemopneumothorax (Tables III and IV).

In each experimental group, mortalities started from the second day following perfusion, and deaths occurred mostly within 3-7 days after the drug perfusion. The survival rate in each experimental group was negatively correlated with the infusion doses (Table IV). In experimental group 4, the 15 tree shrews died within 2-7 days after drug infusion and the survival rate was $0 \%$. Obvious lung congestion was observed during the autopsies and deaths were confirmed to be due to the lethal drug toxicity.

Lung imagings of tree shrews. The results from the chest $\mathrm{X}$-ray examinations conducted on animals in the blank control and solvent control groups at each time point revealed no obvious abnormalities (Fig. 1A and B). In the experimental group, the chest X-ray examination results for tree shrews in the $3 \mathrm{rd}, 7$ th and 11th weeks after perfusion showed focals of high-density shadow spots within the perfusion sites (Fig. 1C and E). We considered these high-density shadows to be the product of lipiodol incomplete metabolism. Pathological studies, performed on the high-density areas, confirmed changes in the bronchial epithelium.

Tumor results of the tree shrews. H\&E staining was conducted on lung tissues collected from tree shrews in each group. We observed no obvious abnormal pathological changes in the bronchial epithelial structure in lung tissues from tree shrews in the blank control and solvent control groups at different time points (Fig. 2A). The results from the H\&E staining conducted on tissues from the experimental groups in the 3 rd week revealed lung tissue congestion. We observed inflammatory changes, hemorrhage and bronchial epithelial exfoliation pathological changes (Fig. 2B and C). In the 5th, 7th, 9th and 11th weeks, we detected mild to severe atypical hyperplasia in bronchial epithelium (Fig. 2D and F), and even some visible early invasive carcinoma (Fig. 2G). Bronchial epithelial changes were 
Table V. Pathological changes detected in lung tissues collected from the animals in the experimental group.

\begin{tabular}{lcccc}
\hline & \multicolumn{2}{c}{ Dysplasia } & Carcinoma in situ \\
\cline { 2 - 4 } Group & Mild & Moderate & Severe & 10 \\
Experimental group 1 & 1 & 1 & 3 & 1 \\
Experimental group 2 & 0 & 1 & 2 & 1 \\
Experimental group 3 & 0 & 1 & \\
\hline
\end{tabular}
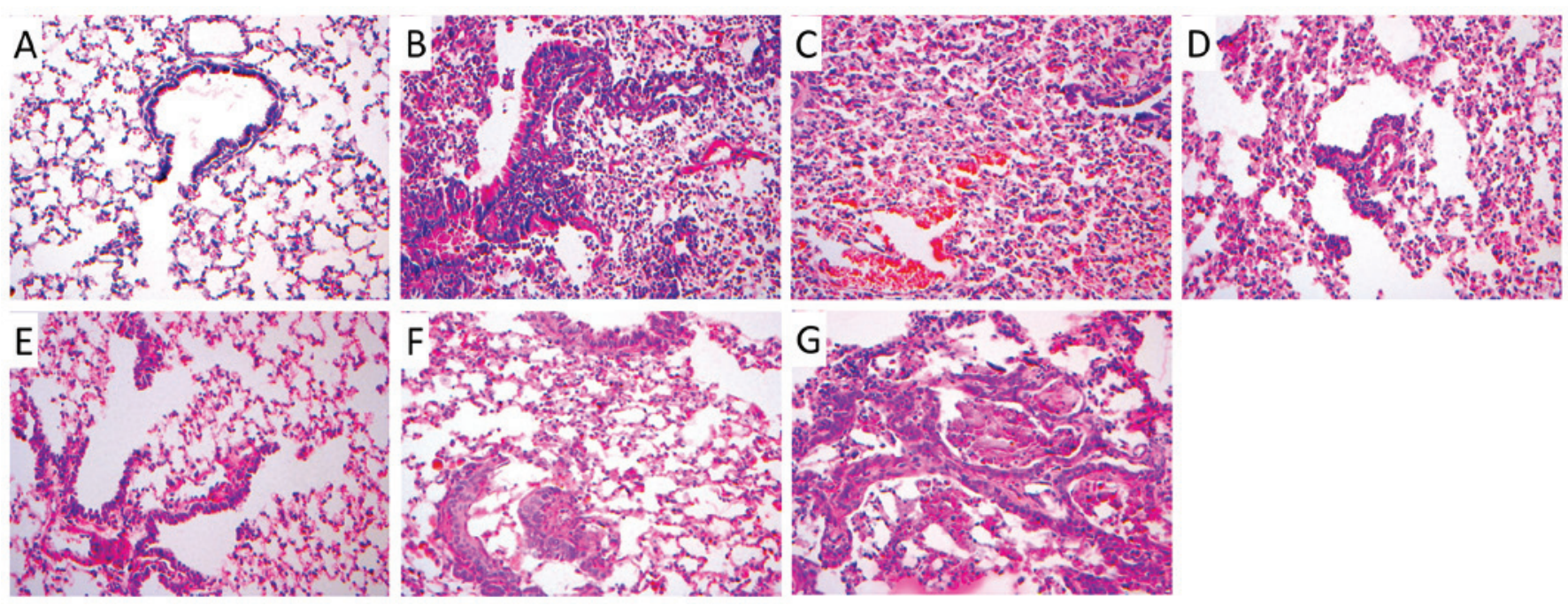

Figure 2. Pathological changes in lung tissues obtained from tree shrews observed under a light microscope, following hematoxylin and eosin staining; magnification, x200. (A) Tree shrews normal lung epithelial structure in the solvent control group in the 11th week. (B) Infiltration of inflammatory cells in lung tissue in the 3rd week (animal in the experimental group). (C) Alveolar hemorrhage, bronchial epithelial and other pathological changes of tree shrews in the experimental group in the 3rd week. (D) Mild atypical hyperplasia of bronchial epithelial in a tree shrew from the experimental group in the 5th week. (E) Bronchial epithelial moderate dysplasia change in tree shrews from the experimental group in the 7th week. (F) Bronchial epithelial dysplasia change observed in tree shrews from the experimental group in the 9th week. (G) Bronchial epithelial carcinoma in situ in an animal from our experimental group in the 11th week.

accompanied by inflammation and haemorrhagic manifestations. In the same lung tissue sections, we detected alterations in all the stages. The occurrence of the pathological alterations observed in lung tissues collected from animals in the experimental groups is shown in Table V. In experimental group 1, 12 tree shrews were identified with bronchial epithelial atypical hyperplasia and 2 with apparent changes in carcinoma in situ.

\section{Discussion}

Mouse, rat, hamster, rabbit and other rodent animal models have been employed for lung cancer studies (1-3). Rodents, to some extent, can simulate human lung cancer occurrence and the development process of lung tumors. However, due to species variation, there is a big difference in living environment, cause of disease and social psychological status between rodent and human $(2,12)$. Currently, there is no ideal animal model for lung cancer and the lack of an ideal animal model is one of the most important obstacles for lung cancer researchers. Thus, it is imperative to identify an effective and accurate model for lung cancer studies.

More evolved animals have more complex organ structures and functions. More complex organs in animals render them closer to human organs $(13,14)$. To develop animal models with full simulation, those animals with closest structure, function and metabolism to human beings should be selected. Tupaia belangeri or tree shrew with some metabolic and anatomic similarities to human has been widely used in biomedical research (4-6). Tree shrews are phylogenetically close to primates and have some primitive primate characteristics. The tree shrew may therefore be regarded as a primate model. In addition, the tree shrew has been considered the intermediary ring connecting the insectivorous and primate taxa (4). Physiological functions of tree shrews are close to those of human, and may therefore be suitable to be used as animal models for human diseases (7-9). Currently, the tree shrew is mainly used for the construction of hepatitis B virus, hepatocellular carcinoma, myopia and the social psychological stress model $(6,14,15)$. To the best of our knowledge, no study has been conducted on the tree shrew as a model for the study of lung cancer.

The rat lung cancer model established by $3-\mathrm{MC}+\mathrm{DEN}$ is the model of choice for investigators working in this field $(9,10)$. Induction of the disease by perfusing rats with ipiodol suspension liquid of 3-MC+DEN leads to morphological changes in the airway epithelium and the development of 
invasive squamous cell carcinoma. The development of squamous metaplasia, atypical hyperplasia, early invasive carcinoma, and extensive infiltration of highly differentiated squamous cell carcinoma may be observed $(9,10)$. In addition, induction of lung cancers may contain highly and poorly differentiated squamous cell carcinoma at the same time. This improved animal model of lung cancer had a high success rate, was cost-effective and had a short induction time. As a single factor induced the experimental animal model, it provided a more suitable method for lung cancer mechanisms, tumor immunity and anticancer treatment studies $(9,10)$.

In the present study, we aimed to develop a suitable animal model for lung cancer research using tree shrews from the Yunnan region in China. Several physiological and structural similarities between this animal and human led to investigation of the possibility of using the tree shrew as our animal model. We successfully induced lung cancer in tree shrews by one-time endotracheal introduction of iodized oil suspension of $3-\mathrm{MC}$ and DEN.

The results of the chest X-ray H\&E staining confirmed those findings. As a result, it is plausible to consider the tree shrew as a new animal model in future lung cancer studies.

\section{Acknowledgements}

The present study was funded by the National Natural Science Fund (nos. U1202224 and 81460278) and the Natural Science Foundation of Yunnan Province (nos. 2013FZ276 and 2014FA048).

\section{References}

1. Westcott PM, Halliwill KD, To MD, Rashid M, Rust AG Keane TM, Delrosario R, Jen KY, Gurley KE, Kemp CJ, et al: The mutational landscapes of genetic and chemical models of Kras-driven lung cancer. Nature 517: 489-492, 2014.
2. Kwon MC and Berns A: Mouse models for lung cancer. Mol Oncol 7: 165-177, 2013.

3. Bach PB: Is our natural-history model of lung cancer wrong? Lancet Oncol 9: 693-697, 2008.

4. Zhang XH, Dai ZX, Zhang GH, Han JB and Zheng YT: Molecular characterization, balancing selection, and genomic organization of the tree shrew (Tupaia belangeri) MHC class I gene. Gene 522: 147-155, 2013.

5. Baldwin MK, Wei H, Reed JL, et al: Cortical projections to the superior colliculus in tree shrews (Tupaia belangeri). J Comp Neurol 521: 1614-1632, 2013.

6. Petruzziello F, Fouillen L, Wadensten H, Kretz R, Andren PE, Rainer G and Zhang X: Extensive characterization of Tupaia belangeri neuropeptidome using an integrated mass spectrometric approach. J Proteome Res 11: 886-896, 2012.

7. Amako Y, Tsukiyama-Kohara K, Katsume A, Hirata Y, Sekiguchi S, Tobita Y, Hayashi Y, Hishima T, Funata N, Yonekawa $\mathrm{H}$, et al: Pathogenesis of hepatitis $\mathrm{C}$ virus infection in Tupaia belangeri. J Virol 84: 303-311, 2010.

8. Wu X, Chang Q, Zhang Y, Zou X, Chen L, Zhang L, Lv L and Liang B: Relationships between body weight, fasting blood glucose concentration, sex and age in tree shrews (Tupaia belangeri chinensis). J Anim Physiol Anim Nutr (Berl) 97: 1179-1188, 2013.

9. Bhattacharya S and Haldar PK: Chemopreventive property of Trichosanthes dioica root against 3-methylcholanthrene-induced carcinogenesis in albino mice. J Environ Pathol Toxicol Oncol 31: 109-119, 2012.

10. Gupta C, Vikram A, Tripathi DN, Ramarao P and Jena GB: Antioxidant and antimutagenic effect of quercetin against DEN induced hepatotoxicity in rat. Phytother Res 24: 119-128, 2010.

11. Thuy TT, Morita T, Yoshida K, Wakasa K, Iizuka M, Ogawa T, Mori M, Sekiya Y, Momen S, Motoyama H, et al: Promotion of liver and lung tumorigenesis in DEN-treated cytoglobin-deficient mice. Am J Pathol 179: 1050-1060, 2011.

12. Rudyanto RD, Bastarrika G, de Biurrun G, Agorreta J, Montuenga LM, Ortiz-de-Solorzano C and Muñoz-Barrutia A: Individual nodule tracking in micro-CT images of a longitudinal lung cancer mouse model. Med Image Anal 17: 1095-1105, 2013.

13. Coppola A and Moshé SL: Animal models. Handb Clin Neurol 107: 63-98, 2012.

14. Miziara ID, Magalhães AT, Santos Md, Gomes EF and Oliveira RA: Research ethics in animal models. Braz J Otorhinolaryngol 78: 128-131, 2012.

15. Wang J, Zhou QX, Lv LB, Xu L and Yang YX: A depression model of social defeat etiology using tree shrews. Dongwuxue Yanjiu 33: 92-98, 2012 (In Chinese). 